\title{
Promoción del uso responsable del agua potable desde los planes educativo-ambiental
}

\section{Promotion of responsible use of drinking water from educational- environmental plans}

\author{
Iris Tibisay Godoy Morales \\ tibisaygodoy@gmail.com \\ Código ORCID: 0000-0002-8742-373X \\ Universidad Pedagógica Experimental Libertador, Miranda, Venezuela
}

\section{RESUMEN}

El uso inadecuado del agua ha ocasionado problemas por falta de cultura ambiental. Esto ha llevado a que el Estado ejecute políticas de racionamiento hídrico y energético. Cuestión que ha afectado a todo el país, en especial a zonas de la Gran Caracas, como son Petare, La Urbina y Maca. Por ello, el propósito de esta investigación fue concienciar a la comunidad estudiantil sisomartiniana, desde el servicio comunitario, sobre la importancia del uso responsable del agua potable, mediante la elaboración de un plan educativo-ambiental. El paradigma utilizado fue socio-crítico, investigación-acción, según los momentos de Teppa (2006): diagnostico; planificación, ejecución y valoración. Las técnicas fueron la observación y la entrevista, los instrumentos guía de preguntas y registro de campo. La contribución fue el proceso consensuado de sensibilización y concienciación no solo de la comunidad estudiantil sino también de la aledaña a la institución, para garantizar la sustentabilidad del recurso hídrico.

Palabras clave: plan educativo; ambiente; concienciación; agua potable; consumo responsable

\begin{abstract}
The inadequate use of water has caused problems due to a lack of environmental culture. This has led the State to implement water and energy rationing policies. Issue that has affected the entire country, especially areas of Greater Caracas, such as Petare, La Urbina and Maca. For this reason, the purpose of this research was to raise awareness in the Sisomartinian student community, from the community service, about the importance of the responsible use of drinking water, by preparing an educational-environmental plan. The paradigm used was sociocritical, research-action, according to the moments of Teppa (2006): diagnosis; planning, execution and evaluation. The techniques were observation and interview, question guide instruments and field registration. The contribution was the consensual process of sensitization and awareness not only of the student community but also of those close to the institution, to guarantee the sustainability of the water resource
\end{abstract}

Keywords: Educational plan; environment; awareness; drinking water; responsible consumption 


\section{INTRODUCGIÓN}

Los acontecimientos que han demarcado la historia expresan que, desde tiempos antiguos, la población tendía a desplazarse en aquellos sectores donde existían recursos hídricos que les permitiera desarrollarse y consolidarse como comunidad, sobre todo en relación con la disponibilidad de terrenos para el cultivo en búsqueda de alimentos $\mathrm{y}$ suministros de agua para los diferentes asuntos de índole doméstico, de abastecimiento y de demás actividades económicas.

En otras palabras, los asentamientos humanos se han organizado a lo largo del tiempo para garantizar así sus necesidades básicas, un ejemplo concreto lo plantea Mourín (2012) cuando señala que hacia el cercano y lejano Oriente, a finales del siglo IV del III milenio a.C., las civilizaciones que se situaron sobre los valles aluviales de los ríos Nilo, Tigres y Éufrates, en los valles fértiles del Ganges e Indo y de los ríos Amarillo y Azul, donde se asentaron las civilizaciones India y China, ya que esta situación geográfica les permitió evolucionar como sociedad, debido fundamentalmente a que la agricultura fue la base productiva para esa época, asociado directamente con el aprovechamiento del agua disponible en los cursos de agua.
En Venezuela, la distribución del recurso para las primeras civilizaciones se situó en áreas cercanas al Lago de Maracaibo y Valencia por el norte, mientras que al Sur y el Oriente se ubicaron a orillas del río Orinoco, asentamientos que respondían a la actividad agrícola predominante, ya que el recurso hídrico constituía la principal herramienta tanto para el desarrollo de los cultivos, como para satisfacer las necesidades básicas de cada comunidad indígena de Los Caribes, Los Timotes y Los Cuicas, entre otros.

De tal manera que estas comunidades se vieron favorecidas por la ubicación geográfica del país, ya que en este continente se localizan las principales cuencas hidrográficas a nivel mundial, que lo colocan en un sitial privilegiado en referencia a la disponibilidad del agua potable para consumo humano a nivel global.

Sin embargo, tal como expresa la Secretaría de Medio Ambiente y Recursos Naturales (2010), dentro de diversas zonas a nivel mundial, en relación con la disponibilidad y calidad de agua "si la existente a nivel local está contaminada y no hay alternativas económicamente viables, el acceso es de hecho imposible. Más de 1000 millones de personas en el mundo carecen de acceso a un agua sana” (p. 4). 
Razón por la cual gran cantidad de personas fallecen debido a enfermedades causadas por aguas infectadas y además existen naciones que invierten gran cantidad de dinero para la potabilización del recurso, lo que ha ocasionado que la población se encuentre en una situación de alto riesgo sanitario y ambiental, asociado en la mayoría de los casos con una gestión inadecuada de los recursos hídricos ocasionando el deterioro progresivo de la calidad de vida.

Al respecto, Navarro (2019) plantea que en el caso particular de Venezuela, los sistemas de agua potable y saneamiento cuentan con infraestructuras robustas, pero severamente deterioradas, los valores de pérdidas físicas se estiman entre $53 \%$ y $75 \%$ de agua producida, perdidas que se generan en la roturas y fugas permanentes en la redes, las cuales son ocasionadas por la falta de mantenimiento, la operatividad inadecuada del sistema y los desgastes de los equipos producidos en el tiempo, hechos que se repiten en otras ciudades del mundo.

Por ello, el Banco Interamericano de Desarrollo -BID- (2019) expresó que la Asamblea General de las Naciones Unidas, reconoció desde el 2010 "el derecho humano al agua $y$ al saneamiento (DHAS), reafirmando que el agua potable limpia y el saneamiento son esenciales para la realización de todos los derechos humanos, según resolución A/RES/64/292” (p. 3).

Hecho que justifica que dentro del Objetivo 6 del Desarrollo Sostenible (ODS), las metas establecen que de aquí al 2030, entre otros aspectos: lograr el acceso universal y equitativo al agua potable a un precio asequible para todos; 6.2: el acceso equitativo a servicios de saneamiento e higiene adecuados para todos y poner fin a la defecación al aire libre, prestando especial atención a las necesidades de las mujeres y las niñas y las personas en situaciones vulnerables; 6.4: aumentar considerablemente el uso eficiente de los recursos hídricos en todos los sectores $\mathrm{y}$ asegurar la sostenibilidad de la extracción y el abastecimiento de agua dulce para hacer frente a la escasez de agua y reducir considerablemente el número de personas que sufren falta de agua.

En consecuencia, tal como lo indica el BID (2019) para "Latinoamérica y el Caribe (LAC), la importancia de la organización comunitaria radica en la cercanía con las necesidades de la población, que construye, gestiona e implementa el servicio" (p. 3), esto con la finalidad de abordar oportuna $y$ adecuadamente los problemas que se puedan suscitar, en relación con la distribución y uso que se le da al agua potable dentro de la comunidad, para contribuir con la sustentabilidad del 
recurso y garantizar la calidad tanto del servicio, como de dicho recurso.

No obstante, la problemática del agua potable, particularmente en Venezuela, se ha acentuado en los últimos años, en virtud de diversos problemas de índole natural y antrópico. En primer lugar, asociado con los problemas de racionamiento, ocasionados por la escasez del recurso, derivado de las condiciones climáticas que ha experimentado el país recientemente.

En segundo lugar, por la falta de mantenimiento de los equipos, aunado al desconocimiento de la población sobre las consecuencias que tiene el uso inadecuado e irresponsable del agua potable, tanto a nivel de la salud para poder satisfacer las necesidades básicas, como a nivel comunitario, por la insalubridad, la mala disposición y almacenamiento del recurso, ya que dichas comunidades no tienen la orientación necesaria para atenuar esta situación de cara a los problemas generados por la falta de gestión y el incumplimiento de las medidas de saneamiento y potabilización del agua.

Sobre la base de estas premisas, surgieron las siguientes interrogantes: ¿qué necesidades de formación tienen los estudiantes Instituto Pedagógico de Miranda José Manuel Siso Martínez (IPMJMSM), para desarrollar la promoción del uso responsable del agua potable en las comunidades?; ¿qué contenido teórico-metodológico debe incluir el plan educativo-ambiental para promover el uso responsable del agua potable desde el IPMJMSM?; ¿cuál ha sido el alcance de la aplicación del programa educativo ambiental para promover el uso responsable del agua potable desde el IPMJMSM, hacia las comunidades adyacentes?

A tal efecto, se planteó la presente investigación desde la Universidad Pedagógica Experimental Libertador, con el propósito de elaborar un plan educativo-ambiental que permita concienciar a la comunidad estudiantil de esa casa de estudios, sobre la importancia del uso responsable del agua potable para garantizar la sustentabilidad del recurso, debido fundamentalmente a que este escenario es clave para apoyar las bases de la educación ambiental, por el potencial que representa la formación de docentes dentro de la transformación de las sociedades hacia una mejor calidad de vida de la población.

Para lograr este propósito, se plantearon los siguientes objetivos específicos, en relación con los estudiantes: diagnosticar las necesidades de formación, para su participación en la promoción del uso responsable del agua potable; elaborar un plan educativo-ambiental considerando los aspectos teóricometodológicos que promuevan el uso 
responsable del agua potable en las comunidades adyacentes al instituto; ejecutar el plan educativo-ambiental para promover el uso responsable del agua potable desde el instituto y valorar la influencia del plan educativoambiental para promover el uso responsable del agua potable desde el IPMJMSM hacia las localidades cercanas a dicho instituto. Tales objetivos se desarrollaron partiendo de la conjunción entre la prestación del Servicio Comunitario, con las competencias que debe tener un egresado de educación, ya que el mismo debe estar formado como promotor comunitario, por tanto, puede atender los problemas asociados con la crisis ambiental, fortaleciendo $y$ enriqueciendo la plataforma teórica que permite ejecutar futuras investigaciones en áreas específicas de las ciencias sociales, incluyendo la educación ambiental y sus buenas prácticas.

\section{MÉTODO}

La investigación se desarrolló bajo el enfoque cualitativo, que según Bogdan (1998) representa "un conjunto de supuestos sobre la realidad, sobre como se conoce, los modos concretos, métodos o sistemas de conocer la realidad, desde el punto de vista ontológico, epistemológico y metodológico” (p. 23). Así, que asumir dicho enfoque permitió observar el escenario, describirlo, analizarlo y comprender la realidad, asociada con el uso que se da al recurso hídrico en localidades adyacentes al instituto, con la finalidad de proponer posibles soluciones a los conflictos generados por el despilfarro, escasez, saneamiento, distribución $y$ racionamiento del agua potable.

Sobre la base del propósito de la investigación, esta se fundamentó en el paradigma socio-crítico aplicado a la educación en la búsqueda de la liberación del hombre, la distribución del poder y de los recursos en la sociedad, enmarcados en un proceso de investigación acción, como una de sus manifestaciones más características.

Al respecto, Kemmis (1984), precursor de la investigación-acción señala que esta en el ámbito educativo constituye:

una forma de indagación
autorreflexiva realizado por
quienes participan (profesorado,
alumnado, o dirección por
ejemplo) en las situaciones
sociales (incluyendo las
educativas) para mejorar la
racionalidad y la justicia de: a)
sus propias prácticas sociales o
educativas; b) su comprensión
sobre las mismos; y c) las
situaciones e instituciones en que
estas prácticas se realizan (aulas
o escuelas, por ejemplo). (p. 3)

En otras palabras, este proceso se asume desde una ciencia crítica, en la cual es permitido que el investigador se incorpore activamente a la investigación, 
con el objeto de trabajar desde el colectivo y de forma sistémica, planificando y ejecutando acciones que resuelven problemas concretos de la realidad de los involucrados en el estudio, a través de un entorno moral, claro, negociado, consensuado $y$ aprobado por todos, para mejorar tanto el sistema educativo y como el sistema socio-cultural, que deriva en la sostenibilidad de los recursos y en una mejor calidad de vida.

De modo que el estudio desarrollado sobre las bases de este paradigma, permitió sistematizar, coordinar $y$ proponer transformaciones conceptuales en los estudiantes participantes dentro del proyecto de servicio comunitario, denominado Gestión del Agua Potable, con la finalidad de promover el mejoramiento continuo del ambiente que el hombre ha deteriorado y del cual hace uso desmedido, limitando la sostenibilidad de los recursos para las generaciones futuras, a la luz de un mundo globalizado y de cara al vertiginoso avance de la ciencia y la tecnología, que en algunos casos ocasiona graves consecuencias en el ambiente.

Desde la investigación acción, el estudio promovió la concienciación, emancipación y empoderamiento de los conflictos asociados con la promoción del uso adecuado del recurso hídrico, a fin de garantizar que las generaciones futuras dispongan de este en las mismas condiciones y calidad.

Por otra parte, es importante destacar que la investigación se desarrolló considerando los momentos propuestos por Teppa (2006):

1. Diagnóstico: conocer las necesidades de formación de los estudiantes del IPMJMSM, para promover el uso responsable del agua potable en las comunidades.

2. Planificación: preparar y organizar las actividades $y$ acciones para elaborar el plan educativo ambiental y abordar desde el proyecto de servicio comunitario denominado "gestión del agua potable" los conflictos generados por esta situación.

3. Ejecución: efectuar las acciones planificadas para solventar los inconvenientes que causa el manejo inadecuado del recurso hídrico en algunas de las comunidades adyacentes al instituto.

4. Evaluación: valorar las acciones realizadas por los estudiantes para promover el uso adecuado del agua en las comunidades.

5. Sistematización: reconstruir las experiencias de todo el proceso de investigación, para fortalecer el plan educativo.

En relación con el procedimiento que acompañó cada uno de estos momentos, 
desde el ámbito de la investigación, vale decir que el mismo se basó en el estudio metódico, detallado y absorto de los conflictos que se han generado en las adyacencias del IPMJMSM en los últimos años por el uso inadecuado del recurso hídrico. Para ello, se realizaron un conjunto de actividades que permitieron obtener y divulgar un conocimiento significativo, a través de la interacción entre los sujetos y provocar cambios sociales para intervenir de manera consensuada en la transformación del comportamiento tanto de los participantes en el proceso, como de la comunidad.

Mientras que la acción se centró en el diseño del plan educativo-ambiental, el cual contempló la sensibilización, concienciación y organización de las acciones sociales en beneficio de promover el uso responsable del agua potable en la comunidad desde el pedagógico.

El escenario planteado para esta investigación correspondió a las comunidades adyacentes al IPMJMSM, especialmente, La Urbina, San José de Petare y Maca, ubicadas en la parroquia Petare del municipio Sucre, Distrito Capital, en las cuales se detectaron conflictos con respecto al uso del agua potable, asociad+as con la distribución, disponibilidad, racionamiento y escasez, por lo cual se consideró relevante tomar a estas comunidades para desarrollar la investigación.

En relación con los participantes de la investigación, estos están constituidos por informantes claves, conformados, de manera directa, por estudiantes de la comunidad Sisomartiniana, de diversas especialidades, cursantes de los últimos períodos, como es el caso de educación física, geografía e historia, preescolar, educación técnica industrial e integral.

Las técnicas empleadas fueron: la observación participante, el análisis documental, las discusiones grupales $\mathrm{y}$ la entrevista estructurada. Los instrumentos: el diario de registro, el cuestionario y el guion de preguntas orientadoras. Para facilitar la recogida de la información se utilizó la grabadora de periodista y la cámara fotográfica.

Es importante acotar que se dio respuesta a cada uno de los objetivos planteados y se fortaleció el plan educativo, para que posteriormente pueda ser aplicado en otros contextos, donde existan problemas asociados con el uso inadecuado del agua potable.

A tal efecto, para realizar el análisis se aplicó la categorización, triangulación, discusión con pares o expertos y transferencia. En el caso de la triangulación se procedió a clasificar, conceptualizar y codificar la información 
a objeto de contrastarla e interpretarla de manera adecuada. Asimismo, se categorizó las incidencias, en atención a la revisión de los sustentos teóricos y al diagnóstico, entendido que las categorías y subcategorías, según Hurtado y Toro (1997), representan:

Uno de los elementos básicos a tener en cuenta es la elaboración y distinción de tópicos a partir de lo que se recoge y organiza la información. Para ello distinguiéremos entre categorías, que denotan un tópico en sí mismo, y las subcategorías que detallan el tópico en micro aspecto. (p. 64)

De tal manera que estas constituyen los aspectos, elementos $y$ factores que pueden asumir diferentes valores, cada vez que son examinados o que reflejan distintas revelaciones según el contexto donde se representa el proceso investigativo. Por consiguiente sobre la base de los objetivos propuestos, se asumieron como categorías y subcategorías, las que se presentan en el Cuadro 1:

Cuadro 1. Proceso de categorización

\begin{tabular}{|c|c|c|}
\hline Categorías & Definición & Subcategorías \\
\hline Formación & $\begin{array}{l}\text { Conocimientos, habilidades y destrezas dirigidos a } \\
\text { la actualización permanente, para un aprendizaje } \\
\text { innovador, organizado y sistemático a objeto de } \\
\text { transformar conceptos, técnicas, valores y } \\
\text { actitudes. (Godoy, 2011) }\end{array}$ & $\begin{array}{l}\text { Conocimientos } \\
\text { Habilidades } \\
\text { Disposición } \\
\text { Valores }\end{array}$ \\
\hline Plan educativo & $\begin{array}{l}\text { Constituye una serie de procedimientos de } \\
\text { carácter pedagógico, basados en valoraciones } \\
\text { continuas, a objeto de ofrecer servicios educativos } \\
\text { que respondan a las necesidades reales de los } \\
\text { problemas concretos de la realidad donde se } \\
\text { desenvuelve el estudiante. }\end{array}$ & $\begin{array}{l}\text { Participación } \\
\text { Acción Social } \\
\text { Práctica educativa }\end{array}$ \\
\hline Alcance & $\begin{array}{l}\text { Elementos que directa e indirectamente definen la } \\
\text { influencia o importancia de los procedimientos } \\
\text { aplicados durante el plan que contribuyen con la } \\
\text { calidad de vida social, familiar y cultural de los }\end{array}$ & $\begin{array}{l}\text { Importancia } \\
\text { Fortalezas } \\
\text { Eficacia }\end{array}$ \\
\hline $\begin{array}{l}\text { Influencia del } \\
\text { plan }\end{array}$ & $\begin{array}{l}\text { Efectos que ha ejercido del plan educativo sobre el } \\
\text { comportamiento de los involucrados en el proceso } \\
\text { de formación en atención al uso del agua potable. }\end{array}$ & $\begin{array}{l}\text { Transformación } \\
\text { Concienciación Impacto }\end{array}$ \\
\hline
\end{tabular}

En correspondencia con la información presentada en el cuadro anterior, las categorías y subcategorías antes propuestas responden tanto a la revisión documental que acompaña la presente investigación, como al diagnóstico efectuado a los involucrados en el proceso, esto con el objeto de facilitar el análisis y la interpretación de la información obtenida. 


\section{RESULTADOS}

Para dar respuesta a los objetivos propuestos en la investigación, el análisis de los resultados se realizó respetando cada uno de los momentos propuestos para su desarrollo, siguiendo la siguiente estructura:

Momento diagnóstico: se basó en detectar las necesidades de formación presentes en los estudiantes miembros de la comunidad del IPMJMSM, en relación con el interés y la motivación manifiesta por ellos al inscribir el proyecto comunitario, como parte de su proceso académico y por lo cual decidieron participar en mismo.

Se pudo interpretar que los estudiantes sólo tenían los conocimientos básicos acerca de la definición del agua potable como recurso indispensable para la vida, además parte de los encuestados manifestó tener poca información acerca de la importancia y conceptualización del recurso, sin embargo saben que esta procede de diversas represas, pero muestran desconocimiento de cuales son y cómo operan.

Además, se pudo apreciar que la mayoría de los miembros encuestados dentro de las localidades seleccionadas (La Urbina, Petare y Maca) desconoce la función de la hidrológica responsable (HIDROCAPITAL), aunado las condiciones que producen la sequía y las razones por las cuales el gobierno nacional y regional aplica constantemente medidas de racionamiento del vital líquido. Adicionalmente, ignoran lo referido al proceso de tratamiento que debe tener el agua para consumo e incluso como debe ser el adecuado almacenamiento en casos de escasez.

En consecuencia, tanto el grupo de estudiantes de la institución, como los miembros de las comunidades, reconocieron la necesidad de realizar charlas, foros, talleres, divulgación de información sobre el tema, en las áreas comunes, campañas educativas, actividades recreativas e incluso el uso de las redes sociales, que les suministre información sobre el tema.

En el caso de los estudiantes, también demostraron, que a pesar de tener poca información sobre el tema, poseen herramientas didácticas y pedagógicas que resultan de fortaleza para la realización de actividades de concienciación en las comunidades, siendo los multiplicadores ideales de este importante tema.

Estas afirmaciones se encuentran recogidas en la Tabla 1 que se presenta a continuación, que muestra algunos de los comentarios emitidos por el grupo de informantes (estudiantes y miembros de la comunidad) en relación al tema, para las subcategorías planteadas dentro de la categoría "Formación”. 
Tabla 1. Comentarios de los informantes para construir la categoría "Formación"

\begin{tabular}{|c|c|c|c|c|}
\hline \multirow{2}{*}{ Informantes } & \multicolumn{4}{|c|}{ Subcategorías } \\
\hline & Conocimientos & Habilidades & Disposición & Valores \\
\hline 1 & $\begin{array}{l}\text { El agua potable es } \\
\text { aquella que puede } \\
\text { ser utilizada para } \\
\text { consumo } \\
\text { humano, porque } \\
\text { es potabilizada }\end{array}$ & $\begin{array}{l}\text { Como participe en el } \\
\text { proyecto de } \\
\text { promotores } \\
\text { comunitarios, } \\
\text { considero que poseo } \\
\text { mejores herramientas. }\end{array}$ & $\begin{array}{l}\text { Tengo la } \\
\text { motivación } \\
\text { porque } \\
\text { considero que } \\
\text { este problema } \\
\text { es de suma } \\
\text { necesidad. }\end{array}$ & $\begin{array}{l}\text { El agua es muy } \\
\text { importante para } \\
\text { el ser humano, } \\
\text { así como los } \\
\text { demás recursos } \\
\text { naturales. }\end{array}$ \\
\hline 2 & $\begin{array}{l}\text { Agua de embalses } \\
\text { sometidas a } \\
\text { diversos procesos } \\
\text { de tratamiento }\end{array}$ & $\begin{array}{l}\text { Dar información es } \\
\text { una cosa y formar es } \\
\text { otra, solo hablar del } \\
\text { tema no garantiza que } \\
\text { los estudiantes } \\
\text { adquieran valores y } \\
\text { actitudes positivas. }\end{array}$ & $\begin{array}{l}\text { Si dictaría } \\
\text { charlas desde } \\
\text { mis horas d } \\
\text { servicio } \\
\text { comunitario }\end{array}$ & $\begin{array}{l}\text { El agua es } \\
\text { importante para } \\
\text { la vida de los } \\
\text { seres vivos. }\end{array}$ \\
\hline 3 & $\begin{array}{l}\text { El agua potable es } \\
\text { la que viene } \\
\text { embotellada o } \\
\text { purificada por los } \\
\text { filtros de casa }\end{array}$ & $\begin{array}{l}\text { Participaría como } \\
\text { apoyo en la realización } \\
\text { de juegos, maquetas, } \\
\text { para los niños hay } \\
\text { mucha variedad }\end{array}$ & $\begin{array}{l}\text { Puedo colaborar } \\
\text { dentro de las } \\
\text { actividades que } \\
\text { realizarán los } \\
\text { estudiantes en } \\
\text { la comunidad }\end{array}$ & $\begin{array}{l}\text { Hay que } \\
\text { informar a todos } \\
\text { para que } \\
\text { quieran el } \\
\text { planeta. }\end{array}$ \\
\hline 4 & Es el agua tratada & Si las tengo & $\begin{array}{l}\text { Si dispongo de } \\
\text { tiempo }\end{array}$ & $\begin{array}{l}\text { Es muy } \\
\text { importante }\end{array}$ \\
\hline 5 & $\begin{array}{l}\text { El agua purificada } \\
\text { con diversos } \\
\text { tratamientos. }\end{array}$ & $\begin{array}{l}\text { Considero que poseo } \\
\text { las herramientas }\end{array}$ & $\begin{array}{l}\text { Asistiría e } \\
\text { invitaría a mis } \\
\text { estudiantes }\end{array}$ & $\begin{array}{l}\text { Es } \\
\text { importantísima } \\
\text { para todo }\end{array}$ \\
\hline 6 & $\begin{array}{l}\text { El agua Natural } \\
\text { es potable }\end{array}$ & Depende de lo que sea & $\begin{array}{l}\text { Iría a los talleres } \\
\text { y charlas }\end{array}$ & $\begin{array}{l}\text { Es vital para } \\
\text { todos }\end{array}$ \\
\hline 7 & $\begin{array}{l}\text { El agua limpia, } \\
\text { hervida o filtrada. }\end{array}$ & $\begin{array}{l}\text { Manejo algunas } \\
\text { herramientas }\end{array}$ & Si asistiría & $\begin{array}{l}\text { Es de gran } \\
\text { importancia }\end{array}$ \\
\hline
\end{tabular}

Sobre la base de estos comentarios, se comprobó la necesidad de intervenir dentro de las comunidades, desde diferentes ámbitos para abordar diversos conflictos ambientales, ya que aunque pareciera existir una mayor conciencia sobre estos problemas, se ha evidenciado el deterioro de las instalaciones hídricas, mal uso del recurso hídrico, exposición de desechos sólidos, poco ahorro energético, entre otros aspectos que se vienen presentando a causa del desconocimiento que posee la comunidad sobre este tema. 
Por consiguiente, resultó de vital importancia ejecutar planes, proyectos $y$ programas que permitan sensibilizar y alcanzar metas importantes en materia ambiental. Así el programa educativoambiental que surge del momento del diagnóstico contempló la sensibilización general de la población, la cual tendrá como principal propósito, tal como expresa Godoy e Hidalgo (2015):

Abordar los problemas
generados por el uso inadecuado
del recurso agua potable y la
protección del ambiente,
acompañada por la creación de
estrategias, instrumentos y
materiales propicios, para la
formación de la comunidad,
orientada a un aprendizaje
técnico-pedagógico que les
permita entre otras cosas,
obtener un aprendizaje
significativo, ser multiplicadores
de la información, concienciar a
la población y a las comunidades
donde se desenvuelven, desde el
conocimiento y el compromiso
parar promover el el
empoderamiento del saber
ambiental y la cultura del
consumo responsable. (p. 56)

En otras palabras, cada uno de estos elementos debe estar contenido dentro del plan educativo, a fin de obtener resultados satisfactorios y optimizar la ejecución del mismo dentro de cada una de las localidades seleccionadas para tal fin.

Momento de planificación: para dar respuesta al objetivo 2 del presente estudio, con relación a la elaboración del plan educativo-ambiental, considerando los aspectos teórico-metodológicos que promuevan el uso responsable del agua potable y tomando como base tanto los comentarios (ver Tabla 2) obtenidos durante la discusión grupal con los informantes clave que participaron en el proceso, así como las incidencias derivadas de la observación, se estableció como primera medida que el plan debía recoger todos estos hallazgos para otorgarle mayor efectividad $y$ dar respuesta a las necesidades de formación y al proceso de concienciación que demanda cada comunidad.

En tal sentido, surgieron diversas subcategorías, tal como se aprecia en la Tabla 2: 
Tabla 2. Incidencias y comentarios de los informantes para la categoría "Plan educativo"

\begin{tabular}{|c|c|c|c|}
\hline \multirow{2}{*}{ Informantes } & \multicolumn{3}{|c|}{ Subcategorías } \\
\hline & Participación & Acción Social & Práctica Educativa \\
\hline 1 & $\begin{array}{l}\text { Pude estimular y } \\
\text { guiar a los } \\
\text { miembros de la } \\
\text { comunidad para } \\
\text { orientar su } \\
\text { iniciativa, } \\
\text { creatividad, } \\
\text { protagonismo y } \\
\text { capacidad de } \\
\text { participación en } \\
\text { beneficio de } \\
\text { resolver este } \\
\text { problema que nos } \\
\text { aqueja tan de } \\
\text { cerca. }\end{array}$ & $\begin{array}{l}\text { Pudimos desarrollar } \\
\text { verdaderos proyectos } \\
\text { sociales desde la } \\
\text { corresponsabilidad, } \\
\text { responsabilidad y respeto } \\
\text { por nosotros y por el } \\
\text { ambiente. A fin de generar } \\
\text { capital social. }\end{array}$ & $\begin{array}{l}\text { La metodología utilizada } \\
\text { con intencionalidad } \\
\text { pedagógica me permitió } \\
\text { consolidar conocimientos } \\
\text { a través de la prestación } \\
\text { de un servicio que generó } \\
\text { múltiples beneficios }\end{array}$ \\
\hline 2 & $\begin{array}{l}\text { Brindar un } \\
\text { escenario de } \\
\text { confianza y } \\
\text { seguridad, que } \\
\text { permitiera la } \\
\text { interacción, para } \\
\text { lograr la } \\
\text { concientización de } \\
\text { la necesidad de } \\
\text { dar un buen uso a } \\
\text { nuestra agua } \\
\text { potable }\end{array}$ & $\begin{array}{l}\text { Reafirmamos la necesidad } \\
\text { de crear escenarios para la } \\
\text { participación activa de los } \\
\text { miembros de la comunidad }\end{array}$ & $\begin{array}{l}\text { Prepararme para trasmitir } \\
\text { un mensaje a personas de } \\
\text { mayor edad, me permitió } \\
\text { mejorar mis estrategias de } \\
\text { enseñanza, para lograr en } \\
\text { ellos la concientización }\end{array}$ \\
\hline 3 & $\begin{array}{l}\text { A pesar de los } \\
\text { contratiempos, } \\
\text { gracias al apoyo } \\
\text { de la comunidad } \\
\text { pudimos realizar } \\
\text { las actividades } \\
\text { planificadas y } \\
\text { concretar la } \\
\text { consolidación de } \\
\text { las mesas técnicas } \\
\text { de agua. }\end{array}$ & $\begin{array}{l}\text { Fue satisfactorio que a ellos } \\
\text { les quedo el aprendizaje } \\
\text { significativo y más por ser } \\
\text { un recurso natural tan } \\
\text { importante }\end{array}$ & $\begin{array}{l}\text { El plan me sirvió de } \\
\text { mucha ayuda en las clases } \\
\text { de educación, ya les fui } \\
\text { dando luego a mis } \\
\text { estudiantes el gran valor } \\
\text { que tiene el agua. }\end{array}$ \\
\hline
\end{tabular}




\begin{tabular}{|c|c|c|c|}
\hline \multirow{2}{*}{ Informantes } & \multicolumn{3}{|c|}{ Subcategorías } \\
\hline & Participación & Acción Social & Práctica Educativa \\
\hline 4 & $\begin{array}{l}\text { Lo que nos } \\
\text { fortaleció como } \\
\text { prestadores de } \\
\text { servicio fue el } \\
\text { trabajo en equipo } \\
\text { en un ambiente, } \\
\text { participativo, } \\
\text { colaborativo y } \\
\text { cooperativo, } \\
\text { donde cada quien } \\
\text { aprovecho sus } \\
\text { potencialidades. }\end{array}$ & $\begin{array}{l}\text { Me gustó tanto haber } \\
\text { trabajado en este proyecto } \\
\text { que en mi comunidad } \\
\text { conformamos una brigada } \\
\text { juvenil comunitaria, que } \\
\text { cuida nuestro Waraira } \\
\text { Repano, ya que la } \\
\text { educación ambiental es } \\
\text { muy importante, cuidar el } \\
\text { ambiente es cuidar la vida. }\end{array}$ & $\begin{array}{l}\text { Trabajar con estudiantes } \\
\text { universitarios me permitió } \\
\text { comprender lo importante } \\
\text { de educar y concientizar a } \\
\text { la población, no importa } \\
\text { las edades, lo importante } \\
\text { es lograr estrategias que } \\
\text { ayuden a obtener su } \\
\text { atención y comprender lo } \\
\text { que se desea impartir }\end{array}$ \\
\hline 5 & $\begin{array}{l}\text { La aplicación del } \\
\text { plan abrió las } \\
\text { puertas para dar } \\
\text { información } \\
\text { referente al agua } \\
\text { potable no solo en } \\
\text { el siso sino en las } \\
\text { comunidades } \\
\text { donde nosotros } \\
\text { hacemos vida. }\end{array}$ & $\begin{array}{l}\text { Se pudo llevar la } \\
\text { información a personas de } \\
\text { diversas edades, que } \\
\text { tuvieron mucha } \\
\text { participación y motivación } \\
\text { en saber sobre el buen uso } \\
\text { del agua para tener un } \\
\text { buen futuro. }\end{array}$ & $\begin{array}{l}\text { Pudimos realizar distintas } \\
\text { actividades como } \\
\text { carteleras, folletos, } \\
\text { carteles, charlas, entre } \\
\text { otras. }\end{array}$ \\
\hline 6 & $\begin{array}{l}\text { Desde mi espacio } \\
\text { considero que } \\
\text { sólo aporte } \\
\text { algunas ideas que } \\
\text { apoyaron la labor } \\
\text { de mis } \\
\text { estudiantes, para } \\
\text { fortalecer su } \\
\text { servicio } \\
\text { comunitario. }\end{array}$ & $\begin{array}{l}\text { No hubo ninguna } \\
\text { afirmación que manifestara } \\
\text { su participación en este } \\
\text { ámbito. }\end{array}$ & $\begin{array}{l}\text { Me pude percatar a través } \\
\text { de las actividades que } \\
\text { realizaron mis estudiantes } \\
\text { que han logrado } \\
\text { desarrollar las habilidades } \\
\text { y destrezas aprendidas } \\
\text { durante la carrera } \\
\text { universitaria. }\end{array}$ \\
\hline
\end{tabular}




\begin{tabular}{|c|c|c|c|}
\hline \multirow{2}{*}{ Informantes } & \multicolumn{3}{|c|}{ Subcategorías } \\
\hline & Participación & Acción Social & Práctica Educativa \\
\hline 7 & $\begin{array}{l}\text { He administrado } \\
\text { varios grupo de } \\
\text { gestión del agua } \\
\text { potable en } \\
\text { servicio } \\
\text { comunitario, } \\
\text { además de } \\
\text { trabajar el tema } \\
\text { en los cursos de } \\
\text { educación } \\
\text { ambiental }\end{array}$ & $\begin{array}{l}\text { En lo personal creo que el } \\
\text { trabajar en una comunidad } \\
\text { es lo más idóneo para } \\
\text { gestionar las situaciones } \\
\text { difíciles encontradas en } \\
\text { ellas y ayudarlos a } \\
\text { soluciones sus verdaderos } \\
\text { problemas desde la } \\
\text { corresponsabilidad social. }\end{array}$ & $\begin{array}{l}\text { En los talleres de } \\
\text { inducción al servicio } \\
\text { comunitario sobre el agua } \\
\text { potable se les proporciona } \\
\text { elementos a los } \\
\text { estudiantes para } \\
\text { desarrollar el plan desde } \\
\text { cada una de sus } \\
\text { especialidades. }\end{array}$ \\
\hline
\end{tabular}

En este orden de ideas, el plan educativoambiental se estructuró sobre la base del consenso entre los participantes, quedando estructurado de la siguiente manera:

\section{Plan educativo-ambiental}

Fundamentación: el plan implicó factores de índole personal, motivacional, conceptual, social, económico, ecológico, metodológico y político. Por tanto, su impacto obedecerá al compromiso individual y colectivo de los involucrados, tomando en consideración la responsabilidad del comportamiento humano en relación al uso que le da al recurso hídrico en todas sus vertientes. Sin embargo, desde la educación se puede lograr un verdadero proceso de sensibilización para la transformación del comportamiento humano, ya que tal como lo expresa Gazzola (2008) "la educación es el camino para canalizar en la sociedad los aportes del conocimiento y utilizarlos con criterios de sustentabilidad" (p. 8).

Por consiguiente, el plan educativo se fundamentó en la una noción de educación ambiental para la sustentabilidad, derivada de la transformación de ideas, opiniones y conceptos y que genera la formación de ciudadanos y grupos sociales responsable de los espacios naturales donde se desenvuelve y a los cuales interviene constantemente para satisfacer sus necesidades y buscar una mejor calidad de vida, mediante la compatibilidad entre el hombre y la naturaleza, sobre la base de una distribución equitativa de los recursos y bienes disponibles.

Justificación: Desde el punto de vista ontológico es una realidad evidente que con el crecimiento desmesurado de la población venezolana y su concentración 
en las principales metrópolis del país, ha aumentado considerablemente el consumo de agua para estas zonas, lo que aunado a la carencia que existe en la construcción de nuevos embalses, ha disminuido la disponibilidad de ésta para consumo. En consecuencia, diversas poblaciones se han visto afectadas por la falta del recurso.

En lo axiológico es un desafío para la educación universitaria para la sustentabilidad alcanzar la concienciación sobre el consumo responsable de recurso hídrico desde la formación de sus integrantes, para que así asimilen una nueva cultura $y$ transformen su comportamiento, en beneficio de una mejor calidad de vida, ya que tal como lo señala Leff (1997) la cuestión ambiental es una problemática social que trasciende el quehacer de las universidades, al reciclaje de profesionales y la refuncionalización de la educación superior.

Desde lo epistemológico, la educación ambiental, no debe ser vista como una rama de la ciencia, sino más bien como el centro de una formación de actitudes favorables para la preservación de la naturaleza, la transformación del conocimiento y la promoción de los cambios sociales, desde la academia y la libertad de pensamiento, Las transformaciones del conocimiento que induce el saber ambiental, mediante la problematización de sus situación real.
De tal manera que el diseño y la ejecución de un plan educativoambiental, que tenga como propósito la concienciación de la comunidad en relación al uso responsable del agua potable, es de suma valoración e imprescindible a la luz de la conservación preservación de los recursos naturales.

Propósito del plan: Concienciar a la comunidad adyacentes al IPMJMSM acerca del uso responsable del agua potable.

Acciones: Las actividades del plan estuvieron organizadas para un promedio de 20 semanas, fueron planificadas desde el consenso entre los estudiantes involucrados en el proyecto Gestión del Agua Potable y las comunidades a abordar.

Momento de ejecución del plan: el plan educativo fue ejecutado a objeto de dar respuesta al objetivo 3 de la presente investigación y elaborado sobre la base del diagnóstico, por tanto, se desarrollaron diversas actividades dirigidas a la adquisición y el fortalecimiento de los conocimientos relacionados con el agua potable.

En primer lugar se realizó una reunión entre la investigadora y los participantes en el proyecto, donde ella les mostró un panorama general acerca de los conflictos generados por la falta de agua 
potable en la actualidad a nivel mundial, nacional, regional y local, destacando los que se presentan en las comunidades aledañas al Siso Martínez. Seguidamente se planificó una serie de visitas a las instituciones del Estado que podrían presentar asesoría y material de interés para proceder a planificar y desarrollar la campaña informativa y divulgativa sobre los temas asociados con el agua potable, su importancia, uso, entre otros aspectos.

En este sentido, bajo la asesoría de la investigadora, el grupo de participantes se dirigió en primera instancia a HIDROCAPITAL, empresa encargada de la prestación del servicio de agua potable en la zona metropolitana, donde se les proporcionó material divulgativo importante para la campaña informativa, que luego se realizó en cada una de las comunidades.

Para obtener información adicional, se procedió a realizar visitas al embalse lagartijo, el cual proporciona agua a las zonas cercanas al instituto, para ver las condiciones en las cuales se encontraba el mismo. $\mathrm{Al}$ respecto es importante señalar que el grupo no tuvo acceso al sitio, ya que el personal de vigilancia informó que éste se encontraba fuera de servicio y estaban prohibidas las visitas. No obstante, se pudo comprobar el estado de abandono de dicho embalse.
Finalmente, se visitó el Ministerio de Ecosocialismo (MINEC), para recabar información de interés. Se facilitó al grupo una serie de materiales, los cuales sirvieron de insumos para la elaboración de las carteleras informativas, trípticos y demás materiales que acompañaron las campañas de concientización de las comunidades.

Adicionalmente, se efectuaron una serie de actividades donde se desarrollaron, entre otras acciones, distribución de trípticos informativos, elaboración de carteleras en algunos colegios de la zona, colocación de afiches e información en lugares de acceso a las comunidades, creación de un grupo en Facebook, denominado Gestión del Agua Potable, realización de charlas, talleres y visitas casa por casa para distribuir material informativo.

Una vez culminadas estas actividades informativas, se pudo notar que los mismos habían obtenido información de interés a través de las publicaciones mostradas por estos medios, lo que les permitió de alguna manera cambiar su forma de pensar con respecto al uso apropiado que se le debe dar al agua potable, incluso algunos manifestaron que tomaron en cuenta algunos tips y lo habían puesto en práctica en sus casas. 
Momento de valoración: En atención al objetivo cuarto de la presente investigación, el cual estuvo orientado hacia la valoración de la influencia del plan educativo-ambiental para promover el uso responsable del agua potable, se pudo apreciar, a través de la observación, que en la medida en que se asume el servicio comunitario bajo la metodología aprendizaje el servicio será más enriquecedor para compartir y crecer como personas, profesionales $y$ ciudadanos de un país que vive momentos sociales particulares que exigen trabajo de todos, según las posibilidades y roles de cada persona.

Además, llevar a la práctica un conjunto de conocimientos y competencias para promover el uso del agua potable en estas comunidades, promovieron la activación de los consejos comunales para la reactivación y activación de las mesas técnicas de agua, con el objeto de atender los problemas generados por el uso, distribución, saneamiento y racionamiento del agua potable dentro de cada una de estas comunidades.

Momento de reflexión: Producto del proceso investigativo se logró meditar acerca de lo indispensable que los estudiantes universitarios reciban una educación de calidad desde los cursos que impregnan el currículo hasta las demás actividades instituciones para así impregnarlos de un gran sentido de responsabilidad ciudadana que les proporcione libertad de pensamiento, autonomía en la toma de decisiones y herramientas para atender la dinámica social actual que se ve reflejada a través de los problemas ambientales que en la actualidad atraviesa la sociedad venezolana.

\section{CONCLUSIONES}

Desde la prestación de servicio comunitario, el educador se convierte en un gran servidor de la comunidad. Además de formar parte también de la misma, se suma la responsabilidad de orientar, escuchar, atender, solucionar, facilitar, sensibilizar y concientizar debido a esa micro sociedad que nosotros los docentes nos encontramos cada mañana en nuestro trabajo profesional como profesor.

El plan educativo fomentó, tanto en la comunidad estudiantil del Siso Martínez como en las que fueron atendidas por los prestadores del servicio comunitario, una mejora significativa en los conocimientos que tenían sobre la importancia del agua potable, consolidándose como entes multiplicadores en materia de uso responsable del agua potable, lo que redundará en atender de manera más eficaz el problema del recurso hídrico desde los hogares, favoreciendo la sensibilización y garantizado la sostenibilidad.

El proceso de sensibilización y concienciación ambiental permitió, mediante la construcción y reconstrucción de conocimientos, generar aprendizajes concretos en la comunidad sobre la interacción entre la sociedad y el ambiente, desarrollando nuevas relaciones, actitudes y comportamientos hacia la naturaleza. 
Se destaca en esta líneas finales que se logró transformar, en algún grado, la mentalidad de los miembros de las comunidades, ya que en la actualidad se ven menos botes y desperdicios de agua, lo que se puede atribuir a la campaña intensiva de concienciación a la fueron sometidos durante el periodo en que se desarrolló la presente investigación.

En definitiva, es de gran eficacia establecer los vínculos necesarios entre la universidad y las comunidades para atender problemas concretos, a fin de buscar, consolidar y aplicar estrategias y respuestas acertadas, ante la crisis ambiental que en la actualidad vive la población venezolana.

\section{REFERENCIAS}

Ander-Egg, E. (2003). Repensando la Investigación-Acción-Participativa.

Comentarios Críticas y Sugerencias. Colección Política, Servicios y Trabajo Social. Buenos Aires-México: Lumen

Banco Interamericano de Desarrollo -BID(2019). Prácticas y saberes en la gestión comunitaria del agua para consumo humano y saneamiento en las zonas rurales de Ecuador. Recuperado de https://publications.iadb.org/publicatio ns/spanish/document/Pr\%C3\%A1cticas _y_saberes_en_la_gesti\%C3\%B3n_comu nitaria_del_agua_para_consumo_huma no_y_saneamiento_en_las_zonas_rurales _de_Ecuador_es.pdf?download=true

Bogdan, R. (1998). Investigación a los Métodos Cualitativos de Investigación. Buenos Aires: Paidós
Gazzola, A (2008). Tendencias de la Educación Superior en América Latina y el Caribe. Servicio de Información y Documentación. IESALC-UNESCO

Godoy, I. e Hidalgo, M. (2015). Plan Educativo-ambiental para uso responsable del agua potable. EAE

Hurtado, I. y Toro, J. (1997). Paradigmas y Métodos de Investigación en tiempos de cambio. Carabobo. Venezuela: Edición Episteme Consultores Asociados C.A

Kemmis, S. (1984). Point-by-Point Guideto Action Reseach for Teacher. Deakin University

Leff, E. (1997). Conocimiento y educación ambiental. Formación Ambiental, 7(17), 19

Moreno, G (2000). Introducción a la Metodología de la Investigación Educativa 2. México: Editorial Progreso

Mourín, J. (2012). Primeras Civilizaciones. Recuperado de http://profesordehistoriajesusmourin.bl ogspot.com/2013/05/primerascivilizaciones-ubicacion.html

Navarro, I. (2019). Boletín técnico informativo N. 3 Evolución histórica del agua potable. Recuperado de https://issuu.com/lara_ignacio/docs/agu a_potable_informacion_basica_

Secretaría de Medio Ambiente y Recursos Naturales (2010). Promotores Ambientales, Recuperado de http:/wwwgobmx/estados/ sinaloa/noticias/page/prpomotoresambientale s.aspx

Teppa, S. (2006). Investigación Acción Participativa en la Praxis Pedagógica. Barquisimeto. Subdirección de Investigación y postgrado. UPEL. Instituto Pedagógico de Barquisimeto 